\title{
La figura del Jefe de Servicio: ¿qué perfil debe de tener?
}

\author{
Vela Navarrete R, Bajo Arenas JM, Martínez Piñeiro JA, Baena González V, Miñana López B, \\ Unda Urzaiz M, Llorente Abarca C. \\ (Resumen y comentarios de la Mesa Redonda celebrada en la Sede de la Asociación Española de Urología el \\ día 10 de abril del 2008, por iniciativa de la Junta Directiva de la Asociación)
}

Actas Urol Esp. 2008;32(7):673-679

\section{RESUMEN}

LA FIGURA DEL JEFE DE SERVICIO: ¿QUÉ PERFIL DEBE DE TENER?

Se prevén grandes cambios sociosanitarios a corto plazo que afectarán seriamente la estructura departamental y jerarquía asistencial del hospital futuro, universitario público o privado. La Asociación Española de Urología se pregunta si en estas circunstancias, en la que la gestión asistencial y economicista del hospital así llamado "industrial" dominará sobre otros aspectos tradicionales de la jerarquía científica, asistencial y docente del jefe de servicio, modificará su imagen, objetivos, funciones, y en definitiva su autoridad. Asímismo cuales han de ser los atributos de esta nueva generación de jefes de servicio. Para ello la A.E.U. convocó una mesa redonda solicitando opiniones y comentarios que se recogen en el texto adjunto.

Palabras clave: Hospitales. Jefe de servicio. Atributos. Varemos.

\section{ABSTRACT}

THE FIGURE OF THE HEAD OF SERVICE (CHAIRMAN): WHAT PROFILE SHOULD HAVE?

Great social-health care changes in the short term have been predicted that will seriously affect the departmental structure and care hierarchy of future hospital, universitary, public or private. The Spanish Association of Urology (AEU) wondered whether in these circumstances, in which the welfare and economic management of the hospital so-called "industrial" will dominate over other traditional aspects of the scientific hierarchy, social welfare and teaching of head Urology of service, will change his image, goals, functions, and ultimately its authority. Likewise which must be the attributes of this new generation of department heads. To this end the AEU call a roundtable requesting opinions and comments which are reflected in the enclosed text.

Keywords: Hospital organization. Chief of the department. Goals and functions.

S están produciendo cambios extraordinarios en todo aquello que afecta a la asistencia médica, desde los principios fundamentales que sustentan los Sistemas de Salud Pública, el acceso de los enfermos a los recursos humanos y materiales del Sistema, la disponibilidad de los proveedores de cuidados preventivos o terapéuticos, médicos, quirúrgicos o de otra naturaleza, y los "modelos de gestión" del complejo y formidable gasto sanitario. Una reciente publicación, precisamente de un urólogo (La santé n'est past un droit, G. Vallancien), con este título tan provocativo "la salud no es un derecho", sino más bien una responsabilidad personal y colectiva, advierte sobre los inconvenientes de la imparable presión política sobre las estrategias sanitarias y sus previsibles consecuencias.

Con la aplicación de nuevas estrategias y modelos de gestión todo está cambiando. Lo percibimos como una consecuencia más de la evolución social, pero lo que aún no está definitivamente dimensionado es su efecto sobre los diferentes actores del proyecto sanitario; enfermos (usuarios o clientes), médicos (proveedores de salud o simplemente trabajadores de la sanidad), gestores (antes gerentes) y sobre todo, sobre el propio Sistema y su inexcusable orden jerárquico, muy especialmente en lo que se refiere a la autoridad asistencial, en aspectos tan fundamentales y básicos como la figura del Jefe de Servicio, sus atributos, funciones y forma de elección. El asunto es mucho más complicado en España porque ya se reconocen variadas formas autonómicas de entender el compromiso sanitario y su aplicación.

Los cambios actuales se producen con extrema rapidez. Es preciso estar vigilantes y preparados. El oficio de Médico es tan absorbente y nuestra ocupación entre el trabajo obligado y el voluntarismo innato, tan excluyente, que hemos pecado en los últimos años, seguramente también debido otros factores 
añadidos, de descuido y negligencia de nuestros derechos laborales. No se explicaría de otro modo que éste sea el país de los médicos interinos. Es preciso reforzar nuestra presencia en aquellas Instituciones que nos representan, pero sorprendentemente con el mismo ideario que hace 100 años (Organización Médica Colegial, Sociedades Científicas, Sindicatos) cuando el resto de la sociedad camina con otro ritmo y por otras autovías. Quizá sea preciso inventar nuevas agencias que sin marginar a los organismos tradicionales, aúnen esfuerzos, definan objetivos y vigilen su cumplimiento. Frente a esta imagen aparentemente desprotegida de la legalidad laboral que debe presidir nuestro ejercicio profesional, tenemos sólidas convicciones de que la clase médica española recibe, tanto en la universidad como en el período postgraduado, una excelente formación profesional, competitiva con cualquier país europeo. Es preciso que este mensaje quede claro ante la sociedad española. Es preciso reconocer también, que el sistema de formación de médicos y residentes (MIR) debe mantenerse en los cauces actuales, con las modificaciones que sean precisas, pero unitario, nacional, no disgregado por influencias autonómicas

Esta mesa redonda se ha desarrollado intencionadamente como elemento descriptivo de lo que está sucediendo, algunas referencias a lo que ha sucedido, junto a un análisis preventivo de lo que puede suceder y no debería suceder

\section{NUEVOS ESCENARIOS. EL HOSPITAL DEPARTAMENTAL Y OTROS MODELOS}

Recordamos que el hospital La Paz fue el primer hospital jerarquizado de la Seguridad Social. Hasta entonces este hospital había sido utilizado por equipos concretos, independientes, que cumplían objetivos, especialmente quirúrgicos, de manera individualizada. La provisión de plazas de jefe de servicio se hizo por concurso oposición, abierto, público, nacional. Ya los hospitales universitarios de aquella época tenían organización departamental con una estructura jerárquica bien definida, que años posteriores fue modificada, incluyéndose nuevos departamentos como el de enfermería.

Tanto entonces como ahora el jefe de servicio podía ejercer sus funciones en diferentes categorías de hospital (Tabla 1); quizás el hospital público
Tabla 1. Categorías de hospitales según función, jerarquía y tipo de gestión

\section{Funciones y jerarquía}

- Hospital Público Universitario: Departamental, Jerarquizado

- Hospital Público: Departamental, Jerarquizado

- Hospital Privado: Equipos de Trabajo

\section{Gestión}

- Gestión propia del sistema público

- Gestión propia del sistema privado

- Gestión privada de hospitales públicos. Nueva Tendencia

universitario sea el más representativo para aplicar los comentarios que se desarrollaron en ésta mesa redonda. Pero no podemos olvidar que existen hospitales públicos no universitarios, cuyo compromiso asistencial es el más importante. Y por supuesto, existen numerosos hospitales privados, en crecimiento progresivo. Concretamente, con datos referidos al 2004, existían en España 470 hospitales privados con más de 50.000 médicos trabajando para este sistema y un crecimiento anual superior al $17 \%$. Claramente ya se advertía en aquella época, como ciertamente ha sucedido, un notable crecimiento de las inversiones en el sistema médico privado (Tabla 2, Fig. 1). Pero lo que nos interesa más para los comentarios que siguen se refiere a la forma de gestión del hospital público que está cambiando de manera rápida, incorporando estrategias novedosas, pero ya utilizadas en otros países. Puede afirmarse que la nueva tendencia es la de "privatizar la gestión de los hospitales públicos", como un modelo para contener el gasto. Sin duda, la privatización de la gestión hospitalaria es el factor que más influencia tiene, y puede tener, sobre la jerarquía asistencial. La autoridad gerencial en este proyecto queda reforzada. Por otro lado, al convertirse el tema económico en la referencia fundamental del compromiso con las autoridades de la sanidad pública (consejerías) todo queda supeditado a las fronteras del presupuesto (con algún beneficio añadido) lo que obliga a un tipo de actuaciones y de presiones fundamentalmente relacionadas con los índices de actividad (no de calidad) que al menos alteran y socavan la autoridad del jefe de servicio en formas muy variadas. El complemento económico ofrecido 
Tabla 2. Características del sistema sanitario público y privado en España*

\section{Servicio nacional de salud \\ - Consolidado y universal \\ - Cubre $80 \%$ servicios sanitarios y $60 \%$ gastos farmacológicos \\ - Gestiona el 7,7\% del producto nacional bruto}

\section{Servicios sanitarios privados}

- Progresivo aumento (>17\% anual)

- 470 hospitales (1 de 4 camas de hospital)

- Gestión del 29\% de todo el gasto sanitario (2,3\% PN bruto 20.000 millones de ?)

\section{Gestión privada en hospital público}

- ¿Fórmula integradora o contención de gasto?

*Datos del 2005

a los médicos en ampliación de jornada puede acallar rechazos o escrúpulos. Y en el caso de especialidades quirúrgicas, el entusiasmo operatorio permite, poco a poco, que el procedimiento sustituya al enfermo. En sus consecuencias últimas el protagonismo del procedimiento ha contribuido a crear lo que se ha llamado la macdonalización de la cirugía o, finalmente, el llamado "hospital industrial". Seguramente es en este escenario en el que la figura de jefe de servicio tiene dimensiones completamente nuevas, distintas a las tradicionales, y su autoridad puede quedar más comprometida (Tabla 3).
Tabla 3. Características asistenciales y de gestión del hospital industrial

Objetivo: asistencial (docencia e investigación asumidas, si obligada, pero no promocionadas).

Hospital no departamental, no jerarquizado, hospital horizontal (puede mantener jerarquías tradicionales pero sin funciones.

Agendas asistenciales individuales, controladas "on line", en hospital informatizado

Modelo de gestión similar a propuestas Health Maintenance Organization

\section{ATRIBUTOS DEL JEFE DE SERVICIO}

Tradicionalmente el jefe de servicio era el que más sabía, el que mejor operaba, el más motivado. $\mathrm{Su}$ autoridad científica y moral se hacía ver en las sesiones clínica, por sus profundos conocimientos de la materia, su experiencia y sus criterios. Pero también en el quirófano, enfrentándose a los casos difíciles o dominando técnicas de difícil aprendizaje. Por extensión, su autoridad se ejercía poniendo orden en el servicio, en temas mayores o menores; dedicación, disciplina, carga laboral, ayudantías, protagonismo quirúrgico, docencia y programa de investigación y hasta el conflictivo momento de fijar el calendario de vacaciones o asistencias a congresos. Su carga administrativa fundamental estaba relacionada con la planificación humana y material de servicio y el cumplimiento de objetivos numéricos (número de consultas de pacientes nuevos, revisiones, número de intervenciones, etc.) o de calidad (incorporación de nuevas técnicas, análisis crítico de morbilidad y mortalidad operatoria, etcétera). Todo ello sin olvidar su compromiso docente e investigador, que generaba exigencias añadidas de esfuerzo y dedicación.

Esta es, a grandes rasgos, la figura del jefe de servicio tradicional en el que dominaban atributos científicos y técnicos sobre los que se asentaba su autoridad y nivel jerárquico. Ciertamente la

FIGURA 1. Esquema del sistema sanitario español. 
aspiración del resto de los miembros del servicio era alcanzar la jefatura, sólo disponible en caso de muerte o jubilación, por lo que hubo que inventar mecanismos de promoción en el propio servicio, creando subespecialidades (unidades de litiasis, de urodinamia, de andrología, etcétera) por medio de las cuales se consiguiera cierto protagonismo, autonomía y rápido prestigio, además de otro nivel en él estatus social, o dependientes de la administración como la famosa "carrera profesional".

En la actualidad, dependiendo del escenario, las exigencias que debe ostentar en su perfil el nuevo jefe de servicio siguen siendo las tradicionales, pero con ciertos añadidos que bien manipulados pueden conducir a resultados muy dispares en el momento de su elección. Puede mencionarse algunas de ellas y sus comentarios pertinentes: Autoridad individual o trabajo en equipo; capacidad de promoción; capacidad de liderazgo; capacidad gestora. Naturalmente habría que definir claramente estas categorías y baremos para medirlas. Ciertamente estos atributos añadidos representan, sin más, los cambios producidos en nuestra sociedad en los últimos años. La autoridad excesiva, vertical, ya no es aplicable ni en la educación a nivel familiar. La amplitud del conocimiento urológico, científico y técnico, es de tal extensión que es imposible que un individuo domine todas las técnicas y todos los procesos, obligando a compartir compromisos y autoridad. Frente a los controvertidos comentarios de los miembros del Servicio, en una patología concreta, ahora se recurre a las guías de actuación que en cierto modo socavan autoridad, soslayan tiempo de reflexión y justifican actuaciones que son recomendables a nivel general, pero quizás no en el individuo concreto que se comenta. Liderazgo, antes ejemplaridad y motivación, es un terminó ampliamente utilizado en los manuales de gestión, pero raramente en la literatura médica, quizás por sus connotaciones negativas, sociológicas y políticas. ¿Capacidad de gestión?; curiosamente cuando se habla de gestión la referencia exclusiva es la económica y en las condiciones actuales los temas de gestión a discutir con la gerencia, en beneficio del servicio, quedan limitados a recursos humanos y materiales. Si se le llama ser un buen gestor a la capacidad de argumentar con razones sólidas la necesidad de incorporar un nuevo miembro de servicio o la de incorporar un nuevo instrumental, el campo de acción queda muy limitado. Desgraciadamente otras ambiciones de mayor envergadura como incorporar nuevos programas al servicio (trasplante renal, litotricia, urodinamia, etc) depende mucho más del presupuesto del hospital que del entusiasmo y buen hacer del jefe servicio. Finalmente, está la condición humana; virtudes inherentes al carácter del individuo hacen las relaciones más o menos difíciles en el propio servicio o con la gerencia del hospital. Preservar la convivencia en niveles agradables no es siempre tarea fácil (Tabla 4).

Tabla 4. Atributos de un jefe de servicio

Sólida formación clínica y quirúrgica

Liderazgo

Capacidad docente

Capacidad de gestión

Conocimientos de investigación

\section{LA FORJA DE UN JEFE DE SERVICIO}

El jefe de servicio que asumía la dirección de un equipo humano numeroso, con un compromiso asistencial importante, tanto en número como por la gravedad de los procesos a tratar, todo lo que implica una inversión empresarial de notable amplitud administrativa y económica, no se improvisa. Se hizo recuerdo del itinerario formativo de jefes de servicio de pasadas generaciones, su dedicación, su entrega total y sacrificios para conseguir la mejor formación, horas de trabajo extras, estudios hasta el agotamiento, renuncias a otras aficiones personales y familiares, viajes y conocimiento de idiomas, para alcanzar finalmente un concurso oposición difícil y extraordinariamente competitivo. Su éxito no significaba necesariamente la consecución de la plaza deseada si no el comienzo de otro itinerario, geográfico, que resultaba en ocasiones extremadamente costoso en consecuencias económicas y familiares. Afortunadamente todo esto está hoy superado pero, de acuerdo con la ley del péndulo, hemos caído en la endogamia hospitalaria, incluso excluyente de competencia regional o autonómica, y en una forma singular de entender el igualitarismo, tanto formativo como jerárquico. La lucha por la excelencia formativa, arriesgando incluso la plaza fija por un año de ausencia (en ocasiones de excedencia), no tiene sentido en un sistema en el que los 
factores de selección ya no buscan al que más sabe, al que mejor ópera, al más motivado, al más creativo, etc.. No podemos afirmar, aún, si esto es bueno o malo, serán precisos más años de observación, pero ciertamente es lo más cómodo para ciertas estrategias administrativas y de gestión. También es cierto que cuando se aplica esta estrategia de selección se incurre en agravios comparativos y en serias desmotivaciones (Tabla. 5).

Tabla 5. Itinerario curricular para la forja de un jefe de servicio

- Sólida formación clínica y quirúrgica, si preciso a nivel internacional.

- Experto comunicador: Presentaciones científicas. Docencia.

- Experto en relaciones humanas. De carácter abierto, amistoso, próximo a la promoción de su entorno y colaboradores.

- Motivado y ejemplar. Liderazgo.

- Conocedor singularidades proyectos de investigación (laboratorio, experimental y clínica) y métodos de evaluación.

- Idiomas. Capacidad de relación hospital, nacional e internacional.

- Gestión. Conocedor modelos gestión hospitalaria.

- Dispuesto a demostrar su preparación y buen hacer con análisis de resultados periódicos e incluso aceptando o solicitando auditorias externas.

\section{NOMBRAMIENTO DE UN NUEVO JEFE DE SERVICIO}

Se señaló la extraordinaria importancia que tienen los mecanismos de selección y nombramiento de nuevos jefes de servicio para preservar la autoridad del mismo. Aquel jefe que llega a su destino en un concurso abierto, público, lo alcanza con autoridad moral reforzada, entre sus iguales y con la gerencia. Por el contrario, el jefe de servicio nombrado por el gerente pocas posibilidades de éxito puede tener en cualquier tema conflictivo. Parece que con nuestra política autonómica se están diseñando 17 formas distintas para nombrar un jefe de servicio, con claro dominio del concepto endogámico, más del propio hospital, que abierto a la autonomía correspondiente.

Las preguntas siguientes se refirieron al papel de las Sociedades científicas y la Comisión Nacional de la Especialidad en los nombramientos de jefe de servicio y a la existencia o no de baremos oficiales para la valoración de los candidatos. Existen actualmente notables irregularidades en los nombramientos.
En algunos casos se demoran temporal o permanentemente o es nombrado un coordinador; en otros casos, es la propia gerencia quien nombra jefe de servicio, incluso como cargo de confianza, sin pasar por ningún comité de valoración; por el contrario, en algunas autonomías, como la andaluza, se sigue reclamando la presencia de un miembro de la sociedad urológica correspondiente para que esté presente en él concurso o en la habilitación del candidato. No existen evidencias de que algún miembro de la Comisión Nacional de Urología haya sido reclamado para alguno de estos concursos, en función de éste cargo y no por otros motivos. En cuanto a los baremos, sin duda existen y son frecuentemente utilizados en los concursos y habilitaciones universitarias. Otra cosa es que sean aplicados en casos concretos.

¿Son necesarios los jefes de servicio? Ciertamente existen hospitales en los que no hay jefes de servicio porque el hospital no es un hospital departamental. Existen jefes de equipo como sucedía en el hospital La Paz antes de su jerarquización. Estos equipos están formados por tres o cuatro miembros, su objetivo es cumplir con actividades pactadas y recibir compensaciones económicas negociadas. Pero este modelo de actividad está fuera del sistema público. En algunos casos estos equipos han funcionado muy bien y han alcanzado prestigio nacional e internacional, pero esta es la excepción. La mayor parte de la mala práctica americana viene de instituciones de esta naturaleza. La figura de jefe de servicio parece necesaria.

¿Debe ser el cargo de jefe de servicio vitalicio? En algunos sistemas sanitarios los cargos, aunque vitalicios, son auditados periódicamente. Es excepcional la pérdida de puesto de jefe de servicio, por ejemplo en el sistema americano, por motivos profesionales o académicos y los casos que todos conocemos están relacionados con fraudes de variada naturaleza. Nombramientos temporales debilitan la autoridad del jefe de servicio y le exponen a manipulaciones muy variadas. No podemos olvidar la lectura laboral de los contratos, más próximos a la interinidad que al compromiso estatutario.

\section{FUNCIONES DEL JEFE DE SERVICIO}

Por unanimidad y en presencia de antiguos y nuevos jefes de servicio, se reivindicó la figura del jefe de servicio presidida por el atributo de la ejemplaridad en el cumplimiento de sus funciones: dedi- 
cación, dominio del campo clínico y operatorio, generador de entusiasmos y motivaciones. La función fundamental de un jefe servicio es el cumplimiento de su compromiso asistencial en el mejor nivel de calidad, a ser posible en el llamado nivel de excelencia. En este compromiso radica su fortaleza y es donde gravita su formación y su esfuerzo. Todas las demás funciones son negociables pero el compromiso asistencial es el que juzga sus aptitudes y actitudes, por el que realmente se valora su trabajo y la calidad de su oficio; por los enfermos, en primer lugar, pero también por los miembros de su equipo y por el propio hospital.

Se insiste mucho en la capacidad de gestión del jefe servicio. La capacidad de gestión debe referirse a que el objetivo previamente señalado, el compromiso asistencial, se realice en ambientes de calidad, de éxitos, de ausencia de complicaciones, de sosiego, de trato humano, de convivencia envidiable, con los recursos humanos y materiales oportunos. Esa es la planificación sobre la que hay que insistir con convicción, frente a gerencias colaboradoras, esquivas o sordas. En la situación actual los otros temas de gestión, fundamentalmente económicos, están siempre presididos por conceptos de reducción del gasto o contención del gasto; diseñe una política de uso de antimicrobianos en profilaxis quirúrgica que reduzca el gasto en antibióticos; diseñe una estrategia quirúrgica de la próstata que no necesite ingreso; etc. Pero resulta que estas propuestas, o algunas de ellas, no son interesantes para la administración porque o no se puede facturar la cirugía prostática si el enfermo no está ingresado un mínimo de 24 horas, o no se puede facturar un proceso de litotricia si el enfermo no esta ingresado, etc. La capacidad de gestión económica de un jefe de servicio podría medirse, en un proceso de contabilidad analítica en el que se supiera con certeza cual es el presupuesto de un servicio de urología concreto, ofreciéndole la oportunidad de la gestión dentro de unos márgenes numéricos de actividades y una participación de los beneficios obtenidos. Pero esta imagen administrativa no se contempla por ahora aunque sabemos de la existencia de contratos singulares, por ejemplo en anestesia, en el que la provisión de servicio se hace no por ampliación de jornada sino en función de objetivos alcanzados (Tabla 6).
Tabla 6. Funciones del jefe de servicio

- Control calidad asistencial médica y quirúrgica aspirando al nivel de excelencia y con permanente valoración resultados

- Cumplimiento compromisos numéricos pactados con la Gerencia y otros indices de actividad hospitalaria

- Gestión recursos materiales y humanos en el mejor escenario posible

- Cumplimiento compromisos docentes e investigadores

- Ambición de progreso. Organización del servicio e incorporación nuevas técnicas con estos fines

- Ejemplaridad en todas sus funciones y dedicación

\section{CONCLUSIONES}

El objetivo de la mesa redonda no era alcanzar conclusiones finales, de consenso, sino recabar opiniones. De todos modos, pareció existir unanimidad en algunos aspectos relacionados con la situación actual, principalmente motivada por el traspaso de la sanidad a las diferentes autonomías, incluyendo algunos comentarios preventivos.

En el itinerario formativo de los actuales y futuros jefes de servicio es importante resaltar el carácter unitario actual del sistema MIR, controlado, en su provisión de enseñanzas conceptuales y habilidades por la Comisión Nacional. Se recuerda que si el primer paso para ser especialista en urología consiste en un examen, a nivel nacional, no debía quedar el urólogo temeroso de futuros exámenes y concursos. Por otro lado, parece también lógico que representantes de la Comisión Nacional o en su representación de las Sociedades Científicas autonómicas, estén presentes en la confección de los baremos de selección y en las comisiones de valoración de concursos o habilitaciones.

$\mathrm{El}$ itinerario formativo de quien aspira a la jefatura de servicio no debe ser muy distante y distinto del tradicional. Aspirar a ser el que domina la materia con mayor profundidad, con criterios ponderados en sus indicaciones médicas y quirúrgicas, con el dominio de las técnicas quirúrgicas más sobresalientes o complejas. En la perspectiva actual se comprende que haya jefes de servicio que han alcanzado prestigio exclusivamente en temas concretos, sin que obstaculice sus aspiraciones su ignorancia por otros aspectos de la urología, que siempre encontraran en el equipo el complemento deseado.

En el mecanismo de selección del jefe de servicio deben dominar criterios profesionales, ya que su 
compromiso fundamental será el asistencial. Otros aspectos tendrán la relevancia necesaria en función de otros objetivos a cumplir por el jefe de servicio, como su capacidad docente, investigaciones realizadas y proyectos de investigación futura, calidad humana y capacidad de convivencia, capacidad de gestión, etc.

Mientras más abierto, público y competitivo sea el nombramiento de un jefe de servicio, mayor será la autoridad con la que alcance su puesto. Una autoridad que le permitirá, con mayor rigor y solvencia, dirigir el servicio y mantener relaciones con los representantes de la administración y gestión hospitalaria en un marco de respeto mutuo. Por el mismo motivo debe estar dispuesto a mostrar su competencia reclamando auditorias u otros elementos de control que valoren la calidad de su actividad.

En cuanto a sus funciones quedan presididas por su capacidad para asumir el reto asistencial, a ser posible en la excelencia. Sus funciones pueden ser valoradas aplicando los índices habituales de actividad hospitalaria pero tampoco debe importarle, e incluso puede solicitar, auditorias externas, comparativas, un instrumento no existente por ahora, que podría ser útil como iniciativa preventiva $\mathrm{y}$ argumento de exigencia para servicios infradimensionados, con reducido personal, o para moderar ofertas asistenciales desmesuradas.

Otras funciones del jefe de servicio tendrán mayor o menor relevancia dependiendo del marco hospitalario en el que trabaja. Concretamente las funciones docentes, investigadoras, académicas, etc, inexcusables en el marco del hospital universitario para cuya valoración existen baremo tradicionales.

Correspondencia autor: Dr. R. Vela Navarrete Servicio de Urología. Fundación Jiménez Díaz Avda. de los Reyes Católicos, 2 - 28040 Madrid Tel.: 915504800

E-mail autor: Rvelan@telefonica.net

Información artículo: Original - Revisión

Trabajo recibido: mayo 2008

Trabajo aceptado: junio 2008 